\title{
Segmentation of Neighboring Organs in Medical Image with Model Competition
}

\author{
Pingkun Yan ${ }^{1}$, Weijia Shen ${ }^{1}$, Ashraf A. Kassim ${ }^{1}$, and Mubarak Shah ${ }^{2}$ \\ 1 Department of Electrical \& Computer Engineering, \\ National University of Singapore \\ 2 School of Computer Science, University of Central Florida, USA
}

\begin{abstract}
This paper presents a novel approach for image segmentation by introducing competition between neighboring shape models. Our method is motivated by the observation that evolving neighboring contours should avoid overlapping with each other and this should be able to aid in multiple neighboring objects segmentation. A novel energy functional is proposed, which incorporates both prior shape information and interactions between deformable models. Accordingly, we also propose an extended maximum a posteriori (MAP) shape estimation model to obtain the shape estimate of the organ. The contours evolve under the influence of image information, their own shape priors and neighboring MAP shape estimations using level set methods to recover organ shapes. Promising results and comparisons from experiments on both synthetic data and medical imagery demonstrate the potential of our approach.
\end{abstract}

\section{Introduction}

Segmentation of anatomical structures from medical images is often the first step in computer aided diagnosis. Further analysis highly depends on the quality of the segmented structures. In recent years, geometric deformable models, or level set methods [1,2], have been applied to medical image segmentation. During the past decade, tremendous efforts have been put into different medical image segmentation applications based on level sets 3. Many new algorithms have been reported to increase the precision and robustness of level set methods.

When segmenting or localizing an anatomical structure, prior knowledge is usually very helpful. The incorporation of more specific prior information into deformable models has received great attention. Several methods of incorporating prior shape information into boundary determination have been developed. Cootes et al. 4 made a breakthrough by constructing statistical shape models using corresponding points across a set of training images for image segmentation. Leventon et al. [5] incorporate statistical shape influence into the evolution process of geodesic active contours [6] by embedding each shapes in the training data set as the zero level set of level set maps. Qu et al. 7] add an enriched speed term incorporating curvature, shape and texture information into the speed function. More recently, Xie et al. [8] utilize both texture and shape priors when 
defining their energy functional and segmentation is achieved through minimizing the functional. Nevertheless, it should be noticed that while most of level set based methods applied in medical image segmentation deal with only one level set and try to incorporate more prior information to achieve robust results, not much attention has been put into either multiple initializations of level sets or the interactions between multiple distinct level sets. Actually, structures to be segmented are usually surrounded by one or more adjacent anatomical structures. Taking the neighboring structure into consideration may contribute to overcoming the four drawbacks of level sets mentioned in [3], such as leaking through gaps, embedding of the objects.

Zeng et al. 9], proposed two coupled surfaces under level set framework to segment cortex from 3D MR images, which is mainly based on the near constant thickness of the cortical mantle. Most recently, Yang et al. [10] introduce neighbor-constrained 3D segmentation using a level set based deformable model. However, the neighboring contours may intersect with each other under some conditions because intersection is not penalized in the modeling process.

In medical images, distinct boundaries may not exist between the organs to enable edge detection of organ boundaries. Furthermore, the boundaries may be blurred and ambiguous due to partial volume effects [11]. These problems make the segmentation of neighboring organs a challenging task.

In this paper, we present our method which introduces interactions between neighboring contours when carrying out the segmentation process. A novel approach is proposed for segmenting multiple neighboring objects using both prior shape information and interactions between models. The idea of introducing model competition is inspired by the observation that the radiologists segment organs with poorly defined boundaries by considering the anatomical neighboring structures. In our algorithm, the contours evolve locally based on both image forces and interactions between neighboring shape models. They are also under the influence of their own shape estimates globally to extract the organ contours.

\section{Segmentation of Neighboring Organs}

\subsection{Model Description}

Consider an image $I$ that has $M$ objects of interest, which may connect with each other and have similar intensities or texture. Due to partial volume effects, the boundaries may be blurred, which renders many segmentation methods to fail. Our method integrates mutual prior shape information and repulsive interaction between evolving neighboring curves into the segmentation process.

In our approach, curves $C_{i}(i=1, \ldots, M)$ are used to approach the contours of the objects. These curves are allowed to evolve simultaneously to segment the image. These curves evolve partly according to image information as in 12. Each object has its own shape estimate $\hat{C}_{i}$ (estimation details are given in Section 2.3), to guide the evolution of the curve $C_{i}$ in segmentation process. To achieve this, the non-overlapping area of regions enclosed by $C_{i}$ and $\hat{C}_{i}$ needs to be minimized. Similarly, the repulsive force between different curves is defined 
through minimizing the overlapping area between them. To make the algorithm robust and with lower complexity, the overlapping areas of curve $C_{i}$ and other objects' shape estimates $\hat{C}_{j}(j=1, \ldots, M$ and $j \neq i)$, instead of other curves $C_{j}$, are considered. The overall energy functional $E$ is defined by

$$
\begin{aligned}
E= & \sum_{i=1}^{M}\left\{\lambda_{1 i} \int_{i n\left(C_{i}\right)}\left|I(x, y)-c_{1 i}\right|^{2} d x d y+\lambda_{2 i} \int_{\text {out }\left(C_{i}\right)}\left|I(x, y)-c_{2 i}\right|^{2} d x d y\right\} \\
& +\sum_{i=1}^{M} \mu_{i} \oint_{Q_{i}} d q+\sum_{i=1}^{M} \nu_{i} A_{i}+\sum_{i=1}^{M} \xi_{i} A\left(\text { NONoverlap }\left(C_{i}, \hat{C}_{i}\right)\right) \\
& +\sum_{i=1}^{M} \sum_{j=1, j \neq i}^{M} \omega_{i j} A\left(\operatorname{overlap}\left(C_{i}, \hat{C}_{j}\right)\right)
\end{aligned}
$$

where $\lambda_{i}, \mu_{i}, \nu_{i}, \xi_{i}$, and $\omega_{i j}$ are real positive parameters. Parameter $\xi_{i}$ controls the influence of its own shape estimate. Parameter $\omega_{i j}$ controls the repulsive force on the $i_{t h}$ object from the $j_{t h}$ object, while $A$ denotes area in 2D or volume in $3 \mathrm{D}$.

\subsection{Level Set Evolution of the Model}

In the level set formulation, contour let $C_{i}$ denote the contour of the $i_{t h}$ object being segmented, which is embedded as the zero level set of a level set map $\Psi_{i}$, i.e., $C_{i}(t)=\left\{(x, y) \mid \Psi_{i}(x, y, t)=0\right\}$. The signed distance transform is used to generate the level set function $\Psi_{i}$ according to contour $C_{i}$. $\Psi_{i}$ is defined to be positive outside $C_{i}$ and negative inside $C_{i}$. Each of the $M$ objects being segmented in the image has its own contour $C_{i}$ and corresponding $\Psi_{i}$.

The energy functional (11) is formulated in level sets using regularized versions of the Heaviside function $H$ and the Dirac function $\delta$, denoted by $H_{\varepsilon}$ and $\delta_{\varepsilon}[12$, respectively.

$$
\begin{aligned}
E= & \sum_{i=1}^{M}\left\{\lambda_{1 i} \int_{\Omega}\left|I(x, y)-c_{1 i}\right|^{2}\left(1-H_{\varepsilon}\left(\Psi_{i}(x, y)\right)\right) d x d y\right. \\
& +\lambda_{2 i} \int_{\Omega}\left|I(x, y)-c_{2 i}\right|^{2} H_{\varepsilon}\left(\Psi_{i}(x, y) d x d y\right\} \\
& +\sum_{i=1}^{M} \mu_{i} \int_{\Omega}\left|\nabla \Psi_{i}(x, y)\right| \delta_{\varepsilon}\left(\Psi_{i}(x, y)\right) d x d y+\sum_{i=1}^{M} \nu_{i} \int_{\Omega}\left(1-H_{\varepsilon}\left(\Psi_{i}(x, y)\right)\right) d x d y \\
& +\sum_{i=1}^{M} \xi_{i} \int_{\Omega}\left\{\left(1-H_{\varepsilon}\left(\Psi_{i}(x, y)\right)\right) H_{\varepsilon}\left(\hat{\Psi}_{i}(x, y)\right)\right. \\
& \left.+H_{\varepsilon}\left(\Psi_{i}(x, y)\right)\left(1-H_{\varepsilon}\left(\hat{\Psi}_{i}(x, y)\right)\right)\right\} d x d y \\
& +\sum_{i=1}^{M} \sum_{j=1, j \neq i}^{M} \omega_{i j} \int_{\Omega}\left(1-H_{\varepsilon}\left(\Psi_{i}(x, y)\right)\right)\left(1-H_{\varepsilon}\left(\hat{\Psi}_{j}(x, y)\right)\right) d x d y
\end{aligned}
$$


where $\Omega$ denotes the image domain. Keeping each $c_{1 i}$ and $c_{2 i}$ fixed and minimizing energy $E$ in (2) with respect to $\Psi_{i}(x, y)(i=1,2, \ldots, M)$, the associated Euler-Lagrange equation for each unknown level set function $\Psi_{i}(x, y)$ is deduced. After parameterizing the descent direction by an artificial time $t \geq 0$, the evolution equation in each $\Psi_{i}(t, x, y)$ is

$$
\begin{aligned}
\frac{\partial \Psi_{i}}{\partial t}= & \delta_{\varepsilon}\left(\Psi_{i}\right)\left\{\lambda_{1 i}\left|I-c_{1 i}\right|^{2}-\lambda_{2 i}\left|I-c_{2 i}\right|^{2}+\mu_{i} \cdot \operatorname{div}\left(\frac{\nabla \Psi_{i}}{\left|\nabla \Psi_{i}\right|}\right)+\nu_{i}\right. \\
& \left.+\xi_{i}\left(2 H_{\varepsilon}\left(\hat{\Psi}_{i}\right)-1\right)+\sum_{j=1, j \neq i}^{M} \omega_{i j}\left(1-H_{\varepsilon}\left(\hat{\Psi}_{j}\right)\right)\right\}
\end{aligned}
$$

\subsection{MAP Shape Estimation}

In order to incorporate the influence of prior shape model to the evolution process as in (3), the shape and the pose of each model must be correctly estimated. Let $\hat{\Psi}_{i}$ denote the estimated curve of the $i_{t h}$ object. At each step of the curve evolution, it is estimated by

$$
\hat{\Psi}_{i \mathrm{MAP}}=\underset{\hat{\Psi}_{i}}{\operatorname{argmax}} p\left(\hat{\Psi}_{i} \mid \Psi_{i}, \hat{T}_{i}, \nabla I\right)
$$

where $\hat{T}_{i}=\left\{\hat{\Psi}_{k} \mid 1 \leq k \leq M\right.$ and $\left.k \neq i\right\}$. To compute the MAP curve, we expand (4) using Bayes' Rule.

$$
p\left(\hat{\Psi}_{i} \mid \Psi_{i}, \hat{T}_{i}, \nabla I\right)=\frac{p\left(\Psi_{i}, \hat{T}_{i} \mid \hat{\Psi}_{i}\right) p\left(\nabla I \mid \Psi_{i}, \hat{T}_{i}, \hat{\Psi}_{i}\right) p\left(\hat{\Psi}_{i}\right)}{p\left(\Psi_{i}, \hat{T}_{i}, \nabla I\right)}
$$

The normalization term in the denominator of (5) can be discarded since it does not depend on the estimated shape of the $i_{t h}$ object. In addition, by assuming that other estimated shapes are independent of each other, we have

$$
\begin{aligned}
p\left(\Psi_{i}, \hat{T}_{i} \mid \hat{\Psi}_{i}\right) & =p\left(\Psi_{i} \mid \hat{\Psi}_{i}\right) \prod_{k=1, k \neq i}^{M} p\left(\hat{\Psi}_{k} \mid \hat{\Psi}_{i}\right) \\
p\left(\Psi_{i} \mid \hat{\Psi}_{i}\right) & =\exp \left(-A\left(\text { inside } C_{i} \text { and outside } \hat{C}_{i}\right)\right) \\
p\left(\hat{\Psi}_{k} \mid \hat{\Psi}_{i}\right) & =\exp \left(-A\left(\text { inside } C_{i} \text { and inside } \hat{C}_{k}\right)\right)
\end{aligned}
$$

The second term in (5) computes the probability of seeing certain image gradients given the current curve and the estimated curves. Since the gradient is a local feature, it is reasonable to assume that this probability does not depend on other estimated curves. Thus, we have

$$
p\left(\nabla I \mid \Psi_{i}, \hat{T}_{i}, \hat{\Psi}_{i}\right)=p\left(\nabla I \mid \Psi_{i}, \hat{\Psi}_{i}\right)
$$




\section{Experimental Results}

In our experiments, we set $\lambda_{1 i}=\lambda_{2 i}=0.2, \mu_{i}=0.05 \cdot 255^{2}$, and $\nu_{i}=0$ so that only parameters $\xi_{i}$ and $\omega_{i j}$ need to be adjusted to balance the influence of self shape prior and model competition.

\subsection{Evaluation of Model Competition by Simulations}

Our method was first applied on a 2D synthetic image which consists of one black arrow composed of a triangle and a rectangle, with no defined boundaries between them. They are of same intensity and Gaussian noise is added. For each object, a training set of 12 sample contours are used. It is difficult to avoid leakage using only prior shape information. Fig. 1] shows an example of severe leakage where the contours of the triangle and the rectangle leak into other's region. In Fig.2, model competition is taken into account where the same parameters are adopted except for $\omega_{12}=\omega_{21}=0.1$. In Fig. 1]and Fig. 2, yellow curves illustrate the initial MAP shape and pose. Green curves illustrate the final MAP shape and pose. Red curves show the initial contours (small red circles) and final contours. In Fig. 2, leakage is avoided by introducing competition between two statistical models.

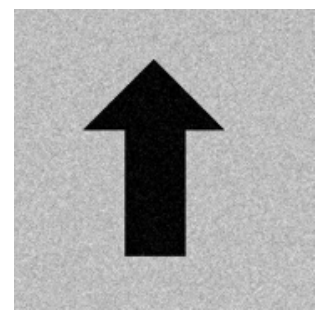

(a)

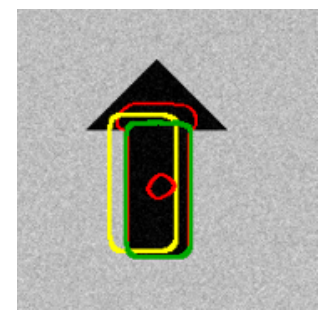

(b)

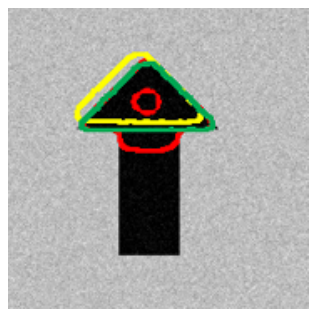

(c)

Fig. 1. Results without model competition $\left(\xi_{1}=\xi_{2}=0.2\right)$ : (a) the original synthetic image, (b) the segmentation results for rectangle, and (c) the results for triangle

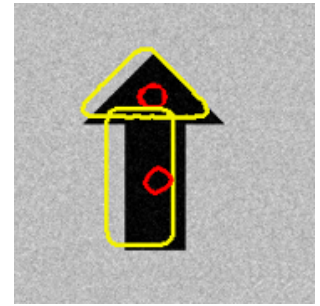

(a)

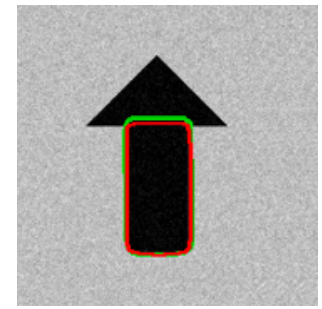

(b)

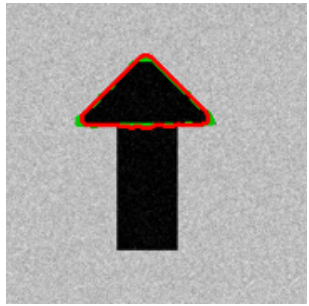

(c)

Fig. 2. Results with model competition. Parameters are the same as in Fig. 1 except $\omega_{12}=\omega_{21}=0.1$ : (a) the initial curves and estimated shapes. (b) the segmentation results of rectangle, and (c) the results for triangle. 


\subsection{Applications to Medical Imagery}

Fig. 3 shows the segmentation of the amygdalae and hippocampus in a 2D MR image. In Fig. 3, the first column shows the initial contours; the second column shows intermediate evolving contours. The third column shows the final segmentation results. The top row, which are the results without any prior knowledge, shows that the two evolving contours become undistinguishable due to extremely poorly defined boundaries and very similar intensities. The middle row shows the results of using only prior shape information but without any model competition (refer to [5] for details). It is clear that the final results, which incorporates shape priors are more meaningful. However, there exist overlapping areas marked by red and green contours due to ill defined boundaries. The overlapping is unavoidable unless the neighboring organ imposes constraint on the evolution of the contour. The third row presents the results of using both shape priors and model competition. Other parameters are the same as for the middle row except
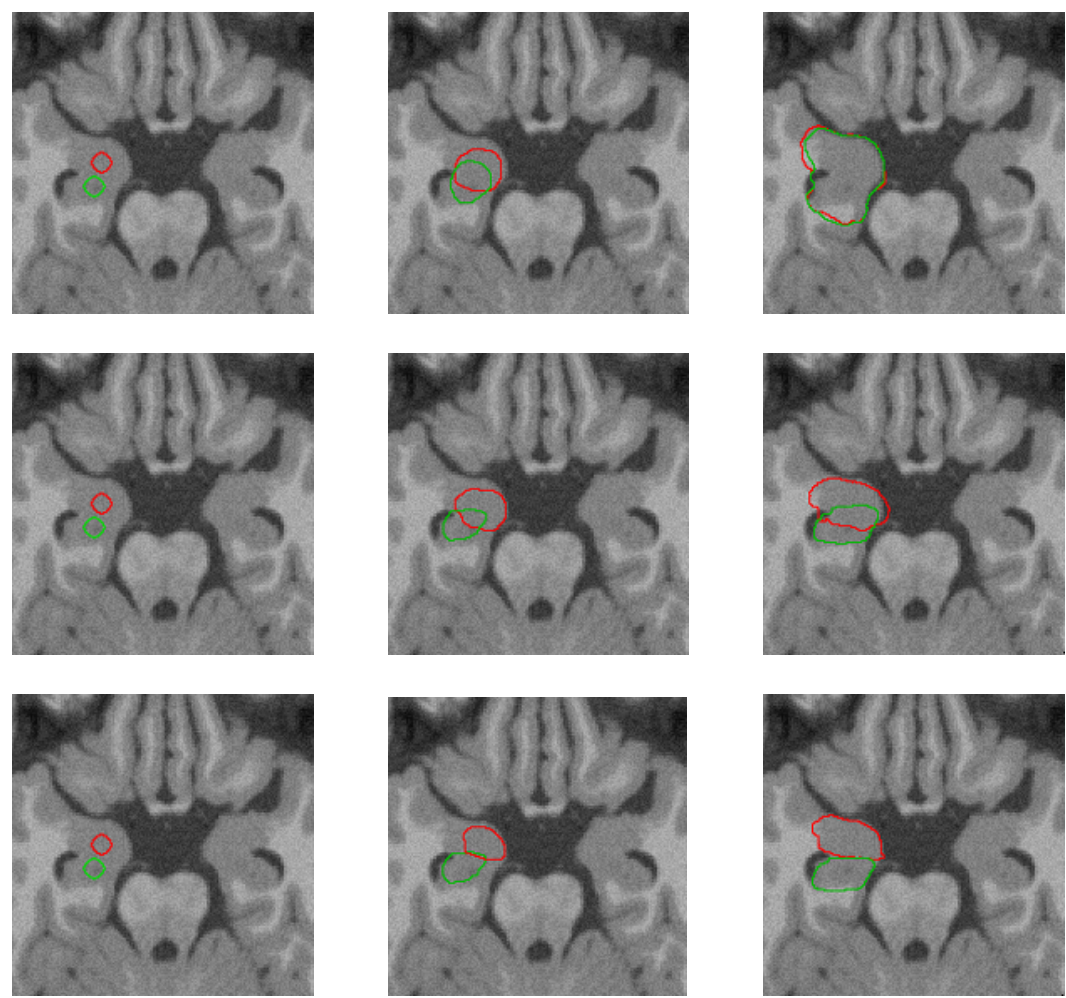

Fig. 3. Three steps in the segmentation of amygdalae and hippocampus in a brain image. (top) Results without prior knowledge. (middle) Results using only self prior shape information with $\xi_{1}=\xi_{2}=0.2$. (bottom) Results using both shape priors and model competition, where $\omega_{12}=0.2, \omega_{21}=0.1$, and other parameters are the same as for the middle one. (The upper structure is object 1 and the lower one is object 2). 


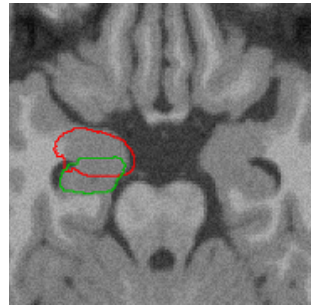

(a)

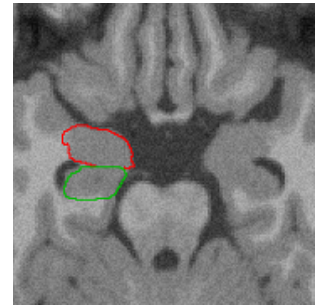

(b)

Fig. 4. MAP shape estimates of the segmentations shown at the middle and bottom rows of Fig. 3 (a) MAP shape estimate with only individual prior shape information. (b) MAP shape estimate with prior shape information and competition between two models.

$\omega_{12}=0.2$ and $\omega_{21}=0.1$. In the final results (column 3 of Fig. 3), the two structures are nicely segmented only when model competition is incorporated (the bottom image in column 3). We also note the overlap is avoided in the corresponding intermediate stage (the bottom image in column 2). Compared with the case of using only prior shape information (middle row), our method does not result in overlapping. Furthermore, in Fig. 4, we can see that introducing competition between models into the multiple object segmentation process will not only prevent overlapping of evolving neighboring contours but also benefit the shape estimation.

In Fig. 4, it can be seen that the final MAP shape estimates are improved after introducing interactions between models. With only shape priors, segmentation processes for each organ are treated independently. When leakage occurs, (as in the second image of the middle row in Fig. 3), an incorrect MAP shape estimate will be obtained, which tends to misguide further evolution of the contour. The final MAP shape estimates are shown in Fig. 4(a). After incorporating interactions between models, evolution of the contours and MAP shape estimates will tend to minimize the overlaps with others as well (see (1) and (6)). Each shape estimate will become less probable when it overlaps with others (see (5) and (6) ). Thus, as shown in Fig. 3] and Fig. 4, allowing model competition will both benefit shape estimation and the contour evolution process to obtain good and robust segmentation results.

\section{Discussions and Conclusion}

In this paper, we focus on how to introduce interactions between neighboring contours and effectively incorporate them into the segmentation process. We have proposed a novel approach for segmenting multiple neighboring objects using prior shape information and competition between models, which intuitively describes the relationship between evolving contours and their own shape estimates, and the relationship between evolving contours and others' shape estimates (see (1)). Without repulsive interaction, our approach degrades to just 
another formulation of active contours with prior shape influence [5]. Our approach is mainly designed for situations where prior shape information is not sufficient to achieve a good and robust estimation due to poorly defined boundaries and very similar intensities. With our method, multiple neighboring objects can be detected simultaneously without suffering from intersection problems and MAP shape estimates can be improved as well. Experiments on synthetic data and MR images show promising results. The proposed method can be applied to $3 \mathrm{D}$ medical image segmentation directly since implicit representations of the curves are used.

\section{References}

1. Osher, S., Sethian, J.A.: Fronts propagating with curvature-dependent speed: Algorithms based on Hamilton-Jacobi formulations. J. Comp. Phy. 79 (1988) 12-49

2. Malladi, R., Sethian, J.A., Vermuri, B.C.: Shape modeling with front propagation: A level set approach. IEEE Trans. Pattern Anal. and Machine Intell. 17 (1995) 158-174

3. Suri, J., Liu, K., Singh, S., Laxminarayana, S., Reden, L.: Shape recovery algorithms using level sets in 2-D/3-D medical imagery: A state-of-the-art review. IEEE Trans. Inform. Technol. Biomed. 6 (2002) 8-28

4. Cootes, T.F., Taylor, C.J., Cooper, D.H., Graham, J.: Active shape models - their training and application. Comput. Vision Image Understand. 61 (1995) 38-59

5. Leventon, M.E., Grimson, W.E.L., Faugeras, O.: Statistical shape influence in geodesic active contours. In: IEEE CVPR. Volume 1. (2000) 316-323

6. Caselles, V., Kimmel, R., Sapiro, G.: Geodesic active contours. Int. J. Computer Vision 22 (1997) 61-79

7. Qu, Y., Chen, Q., Heng, P.A., Wong, T.T.: Segmentation of left ventricle via level set method based on enriched speed term. In: MICCAI 2004. (2004) 435-442

8. Xie, J., Jiang, Y., Tsui, H.T.: Segmentation of kidney from ultrasound images based on texture and shape priors. IEEE Trans. Med. Imag. 24 (2005) 45-57

9. Zeng, X., Staib, L.H., Schultz, R.T., Duncan, J.S.: Segmentation and measurement of the cortex from 3-D MR images using coupled surfaces propagation. IEEE Trans. Med. Imag. 18 (1999) 927-937

10. Yang, J., Staib, L., Duncan, J.: Neighbor-constrained segmentation with level set based 3-D deformable models. IEEE Trans. Med. Imag. 23 (2004) 940-948

11. Pham, D.L., Bazin, P.L.: Simultaneous boundary and partial volume estimation in medical images. In: MICCAI 2004. (2004) 119-126

12. Chan, T.F., Vese, L.A.: Active contours without edges. IEEE Trans. Image Processing 10 (2001) 266-277 